\title{
RNA editing of protein sequences: A rare event in human transcriptomes
}

\author{
CLAUDIA L. KLEINMAN, ${ }^{1}$ VÉRONIQUE ADOUE, and JACEK MAJEWSKI \\ Department of Human Genetics, McGill University-Genome Quebec Innovation Centre, Montreal, Quebec H3A 1A4, Canada
}

\begin{abstract}
RNA editing, the post-transcriptional recoding of RNA molecules, has broad potential implications for gene expression. Several recent studies of human transcriptomes reported a high number of differences between DNA and RNA, including events not explained by any known mammalian RNA-editing mechanism. However, RNA-editing estimates differ by orders of magnitude, since technical limitations of high-throughput sequencing have been sometimes overlooked and sequencing errors have been confounded with editing sites. Here, we developed a series of computational approaches to analyze the extent of this process in the human transcriptome, identifying and addressing the major sources of error of a large-scale approach. We apply the detection pipeline to deep sequencing data from lymphoblastoid cell lines expressing ADAR1 at high levels, and show that noncanonical editing is unlikely to occur, with at least $85 \%-98 \%$ of candidate sites being the result of sequencing and mapping artifacts. By implementing a method to detect intronless gene duplications, we show that most noncanonical sites previously validated originate in read mismapping within these regions. Canonical A-to-G editing, on the other hand, is widespread in noncoding Alu sequences and rare in exonic and coding regions, where the validation rate also dropped. The genomic distribution of editing sites we find, together with the lack of consistency across studies or biological replicates, suggest a minor quantitative impact of this process in the overall recoding of protein sequences. We propose instead a primary role of ADAR1 protein as a defense system against elements potentially damaging to the genome.
\end{abstract}

Keywords: RNA editing; RNA-Seq; ADAR; short read mapping; high-throughput sequencing

\section{INTRODUCTION}

Transfer of genetic information from DNA to RNA is not always perfect. RNA recoding occurs as a result of transcriptional noise as well as of specific post-transcriptional processes, such as RNA editing. Until recently this mechanism that alters RNA sequences at specific sites was considered relatively rare in human cells, mainly restricted to brain-specific substrates and repetitive regions of the genome (Bass 2002). During the last years, however, the development of bioinformatics prediction tools (Levanon et al. 2004) started to challenge this view, followed by the application of deep-sequencing approaches, which allow the study of this phenomenon at a genome-wide scale ( $\mathrm{Li}$

Abbreviations: SNP, single nucleotide polymorphism; gDNA, genomic DNA; ADAR, adenosine deaminase acting on RNA; LCL, lymphoblastoid cell line

${ }^{1}$ Corresponding author

E-mail claudia.kleinman@mcgill.ca

Article published online ahead of print. Article and publication date are at http://www.rnajournal.org/cgi/doi/10.1261/rna.033233.112. et al. 2009b, 2011; Bahn et al. 2011; Ju et al. 2011; Rosenberg et al. 2011; Peng et al. 2012; Ramaswami et al. 2012). Current estimates range from several hundred ( $\mathrm{Li}$ et al. 2009b) to several thousand (Levanon et al. 2004; Bahn et al. 2011; Ju et al. 2011; Li et al. 2011; Peng et al. 2012; Ramaswami et al. 2012) edited sites throughout the genome, suggesting that RNA editing is an abundant process contributing greatly to complexity at both the transcriptome and the proteome levels. Moreover, a large number of sequence changes that are not explained by any of the two known editing mechanisms in humans have been reported in these studies (Bahn et al. 2011; Ju et al. 2011; Li et al. 2011; Peng et al. 2012). If confirmed, this would imply the existence of widespread, highly specific novel mechanisms of RNA recoding, with broad potential implications for our understanding of gene expression.

However, large-scale approaches based on the alignment of sequencing data, especially ESTs and short sequencing reads, are highly susceptible to experimental noise. Patterns of mismatches between DNA and RNA sequences, interpreted as RNA-editing sites, are also generated by genotyping 
errors, sequencing errors, and read mismapping (Schrider et al. 2011; Kleinman and Majewski 2012; Lin et al. 2012; Pickrell et al. 2012). Certain regions of the genome are particularly challenging: repetitive regions (Brawand et al. 2011; Garber et al. 2011; Schrider et al. 2011; Treangen and Salzberg 2012), exon-exon junctions, and positions associated with sequence-specific errors (Meacham et al. 2011; Nakamura et al. 2011). Consequently, published estimates of editing throughout the genome vary by orders of magnitude, depending on the stringency levels used to remove false positives and the coverage and quality of the sequencing data. Given the conspicuous role that genomic sequencing plays in biomedical research today, based on the assumption that genotype is sufficient to accurately predict phenotype, it is essential to determine the actual extent of sequence recoding that takes place during gene expression. This is particularly true for exonic and coding sequences, since they bear important implications for human health and disease.

RNA editing has been shown to occur in a broad range of species, including animals, plants, fungi, protists, and viruses (Knoop 2010). Several types are known, differing considerably in their frequency, molecular mechanisms, and species distribution. In some cases, such as trypanosomatid mitochondria, editing is so extensive that transcripts may no longer be recognized by their similarity to the genomic sequence (Feagin et al. 1988; Bhat et al. 1990). In humans, only two editing types have been described: citidine to uracil (C-to- $\mathrm{U}$ ) and adenosine to inosine (A-to-I) conversions. C-to- $\mathrm{U}$ changes, mediated by the APOBEC enzyme family, have been observed in vivo only in a small number of genes (Rosenberg et al. 2011). A-to-I editing, on the other hand, is the most frequent and well-studied type in mammals. Two members of the adenosine deaminases acting on RNA (ADAR) protein family mediate the conversion of adenosine to inosine in double-stranded RNA, which is later recognized as a guanosine by the cellular machinery. Although the catalytic domain of both ADAR enzymes is highly similar, ADAR1 and ADAR2 have different expression patterns, target genes, and phenotypes obtained in loss-of-function experiments. ADAR2 protein is responsible for the editing of several essential genes in the central nervous system (Sommer et al. 1991; Lomeli et al. 1994; Burns et al. 1997; Huang et al. 2012) and, hence, knockout mice die soon after birth by neurological malfunction (Higuchi et al. 2000) Notably, genomic replacement of a single edited site in a target gene (glutamate receptor GluA2) results in a complete rescue of the ADAR2 null phenotype (Higuchi et al. 2000). The physiological role of ADAR1, on the other hand, is less well established. It is constitutively expressed, with one of its isoforms driven by an interferon inducible promoter (George and Samuel 1999). ADAR1 deficiency results in embryonic death, linked to defects in hematopoiesis and hepatocyte survival. No known target or targets have been identified that may account for the ADAR1 null phenotype despite great efforts to find them, suggesting that the main physiological role of ADAR1 could be unrelated to the selective editing of specific sites. Genome-wide analyses of editing patterns in cells expressing ADAR1 are a valuable tool to assess if this is indeed the case.

In the work presented here, we thoroughly analyzed the extent of RNA recoding in the human transcriptome, with a particular focus on nonrepetitive exonic sequences in cells expressing ADAR1 at high levels. First, we compared transcriptome-wide sequencing data with genome sequencing data of lymphoblastoid cells originating from the same individual. We screened for sites where RNA sequence differs from the corresponding DNA sequence, developing computational tools to address the major sources of errors of a high-throughput sequencing approach: systematic sequencing and alignment artifacts, misalignments around repetitive regions and splice junctions, and missing genotype information. In order to discard any effect specific to our pipeline, cell type, or experimental conditions, we also compiled the sites reported by previous human transcriptome sequencing studies ( $\mathrm{Li}$ et al. 2009b, 2011; Bahn et al. 2011; Ju et al. 2011; Peng et al. 2012). We show that noncanonical editing is unlikely to be real, with at least $85 \%-98 \%$ candidate sites originating in technical artifacts. As for canonical A-to-G sites, very few were located in exons, particularly in coding regions, where the validation rate also dropped. Instead, $>90 \%$ of canonical A-to-G changes occurred in repetitive regions, with a very high enrichment (25-fold) in Alu sequences. Finally, we find almost no overlap of sites across studies, tissues, or biological replicates, suggesting that selective RNA editing of specific sites seems, in the conditions and cell types studied here, not essential for cell survival.

\section{RESULTS}

\section{Detection of editing sites genome wide}

We developed a comparative screening approach for identifying sites with differences between the RNA and corresponding DNA sequences based on the widely used SNP calling algorithms (Fig. 1). The major features that distinguish our framework from other published methods are that (1) genomic SNPs are called with the same settings used for calling RNA-Seq SNPs; thus, genomic DNA alignments are used not only for genotyping but also for detecting sites prone to sequencing and mapping errors; (2) we identify genomic regions where it is not feasible to detect editing sites using short read sequencing: intronless retrocopies of genes and unmappable segments, and (3) we incorporate a set of stringent filters to remove false positives that consider, among other things, positional and directional biases of alternative bases along the sequencing reads.

We applied the framework to two transcriptome data sets (Lalonde et al. 2011) of B-cells from unrelated individuals, 


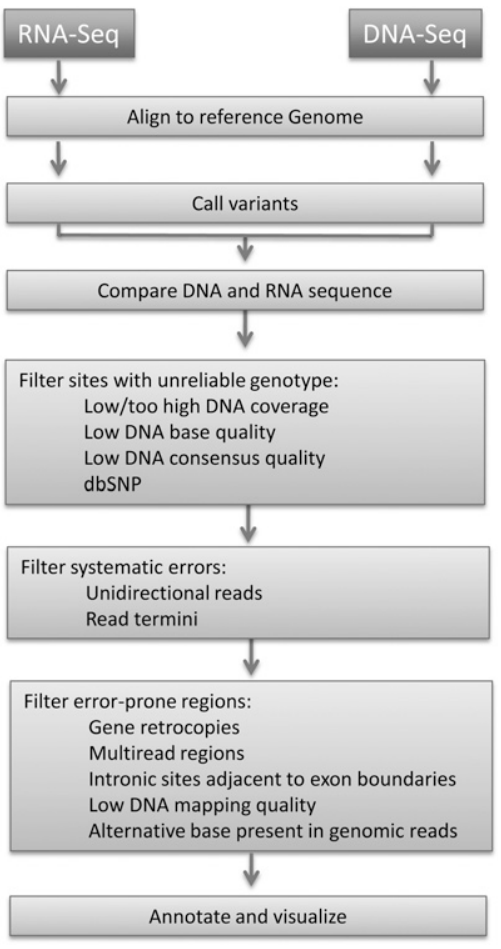

FIGURE 1. Flowchart of the detection method.

for which genomic data $(40-60 \times)$ had been generated in the framework of the pilot 2 study of The 1000 Genomes Project Consortium (2010). Some of the previous transcriptome approaches for identifying RNA-DNA mismatches (Ju et al. 2011; Li et al. 2011) used a large number of individuals, assuming that a recoding event detected in several samples is unlikely to have been generated by stochastic error. However, systematic sequencing and mapping errors, which depend on the particular genomic location and are significantly frequent (Meacham et al. 2011), can produce artifactual DNA-RNA differences recurring in multiple individuals (Kleinman and Majewski 2012; Lin et al. 2012; Pickrell et al. 2012). In contrast, we performed the analysis in samples with high-coverage data both for RNA and DNA sequences, so that genomic DNA (gDNA) alignments are used for filtering sites with unreliable genotype and sites where the alternative base is also seen in the DNA sequence (Fig. 1).

The editing patterns observed at first inspection in our samples are consistent with the expression levels observed for the corresponding ADAR enzymes.
$A D A R$ gene was highly expressed, while ADARB1 (encoding ADAR2 protein) showed a very low expression level. Accordingly, known ADAR2 target sites, such as CYFIP2 (Nishimoto et al. 2008; Riedmann et al. 2008; Horsch et al. 2011) or KCNA1 (Horsch et al. 2011), showed no evidence of editing. Given the high expression level of $A D A R$, we expect A-to-G changes to occur frequently in our samples. C-to-U editing, on the other hand, is much less widespread, and the known enzymes involved showed no detectable expression; the number of $\mathrm{C}$-to- $\mathrm{U}$ changes expected is thus lower. The last category of change that we analyze in this work is the noncanonical nucleotide changes: all other sequence changes not explained by any known biological process. Are these changes real, or the result of technical errors? How do they impact exons and coding regions?

After calling variants with standard quality filters (coverage, mapping, and base quality), a large number of sites with DNA-RNA mismatches are found. A large proportion of these differences, however, is explained by technical artifacts, in particular for noncanonical editing types. Table 1 shows the proportion of candidate editing sites, genome wide, filtered at each one of the main steps of the pipeline. First, note that the majority of noncanonical candidate sites $(65 \%)$ are found in intronic sites immediately adjacent to exon boundaries: 18 times more than expected by chance. For some of the nucleotide changes (G-to-C, G-to-T, A-to-T, T-to-A), this number is higher than $75 \%$ (Supplemental Table 1). These very short segments (6 nt long) are particularly prone to alignment errors in high-coverage samples, since a number of reads will necessarily span splice junctions and bear a short overhang that cannot be accurately aligned (Supplemental Fig. 6). In contrast, for A-to-G changes, the majority of which are most likely produced by a true
TABLE 1. Number of mismatches observed between RNA sequence and corresponding DNA sequence, and proportion of sites found at each major filtering step of the detection pipeline in sample NA12891

\begin{tabular}{|c|c|c|c|}
\hline \multicolumn{2}{|c|}{ Initial DNA-RNA mismatches } & $\begin{array}{l}\text { A-to-G } \\
20,380\end{array}$ & $\begin{array}{c}\text { Noncanonical } \\
57,469\end{array}$ \\
\hline \multicolumn{4}{|l|}{ Filters } \\
\hline A & Intronic sites adjacent to exons & $14 \%$ & $65 \%$ \\
\hline B & Systematic error & $16 \%$ & $37 \%$ \\
\hline C & dbSNP & $8 \%$ & $10 \%$ \\
\hline $\mathrm{D}$ & Retrogenes & $7 \%$ & $7 \%$ \\
\hline $\mathrm{E}$ & Multicopy & $6 \%$ & $5 \%$ \\
\hline \multicolumn{2}{|l|}{$A, B, C, D, E$ combined } & $36 \%$ & $85 \%$ \\
\hline & Dense sites & $44 \%$ & $44 \%$ \\
\hline & Alu regions & $85 \%$ & $38 \%$ \\
\hline & Other repetitive regions & $9 \%$ & $34 \%$ \\
\hline \multicolumn{2}{|c|}{ Sites that passed the filters } & $1.5 \%$ & $2.1 \%$ \\
\hline \multicolumn{4}{|c|}{ Nonrepetitive regions, all filters combined } \\
\hline \multicolumn{2}{|c|}{ Genome-wide } & 312 & 1,190 \\
\hline \multicolumn{2}{|l|}{ In exons } & 70 & 401 \\
\hline
\end{tabular}


biological process, only $14 \%$ are located in these regions. Second, other systematic errors producing biased distributions in the direction of sequencing reads (unidirectional reads) or in the position of the edited site along the read (i.e., preferentially found in the first and last bases of a read) also account for a high proportion of the noncanonical candidate sites (37\%). The number of canonical A-to-G changes falling into this category (16\%), on the contrary, is close to what is expected by chance (Kleinman and Majewski 2012). Third, $10 \%$ of candidate sites correspond to sites present in dbSNP, 10 times more than expectations (Kleinman and Majewski 2012). Since there is no reason for an enrichment of edited sites in polymorphic positions, this indicates that even when using high-coverage DNA sequencing data, genotyping errors are still present in these samples, and are thus confounded with edited sites.

Finally, we identified regions in the genome where current methods of short-read sequencing are not adequate to detect RNA editing (see Materials and Methods for details regarding the detection algorithms). On one hand, multicopy segments: segments of the reference genome that a priori produce reads mapping to multiple locations. It is not possible to assign with confidence the correct location of a variant in these segments. On the other hand, retrogenes consisting of intronless copies of transcribed genes (Fig. 2; Supplemental Fig. 5). When a read spans a splice junction of the actively transcribed gene, the commonly used exon first approaches to reconstruct the transcriptome tend to prefer the (incorrect) alignments with one or a few mismatches over the (correct) gapped alignments that correspond to spliced transcripts. As a result, editing sites are incorrectly assigned to the pseudogene location. Furthermore, traditional Sanger sequencing cannot resolve the ambiguity in most cases, since the same artifactual patterns are obtained by misamplifications during the PCR step (Fig. 2). We detected 4848 distinct segments in the reference genome that belong to this class, accounting for $7 \%$ of the DNA-RNA mismatches observed in our samples (Table 1). Previous studies reported validation experiments of noncanonical editing sites with a false-positive rate of $49 \%$ (Bahn et al. 2011; Ju et al. 2011; Peng et al. 2012). In order to further investigate the existence of noncanonical editing, we compiled a data set of noncanonical editing sites previously reported as validated by Sanger sequencing, and found that at least $73 \%$ of these sites overlap intronless retrocopies of genes or segmental duplications (Supplemental Table 2). Since Sanger sequencing is not sufficient to validate these regions, the actual validation rate of noncanonical sites to date would be at most $13 \%$. As shown by this experiment alone, much more evidence is needed in order to prove the existence of novel mechanisms of RNA recoding.
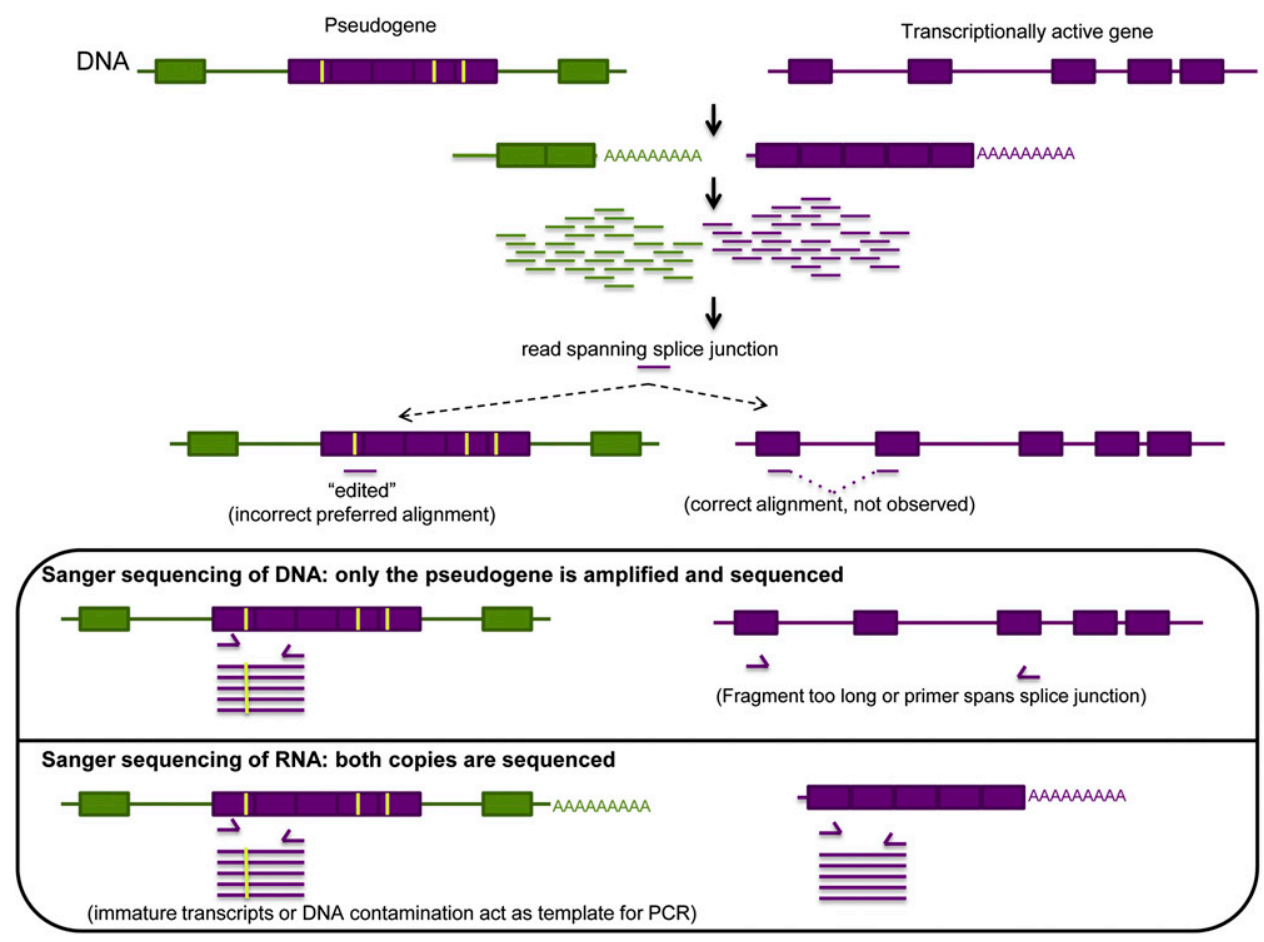

FIGURE 2. Pseudogenes lacking introns are a source of false positives. Retrotransposition of processed mRNAs is a common source of sequence duplication in the human genome. "Exon-first" approaches to transcriptome reconstruction tend to have a high rate of misalignments around these regions, producing sites that are incorrectly interpreted as edited sites. Validation by Sanger sequencing usually does not resolve the ambiguity, since it requires a PCR step, where traces of unprocessed transcripts or DNA contaminations can act as templates to reproduce the artifact obtained when using RNA-seq. Yellow vertical lines represent substitutions, very common in pseudogenes. 
Combining all of the filters described above, $85 \%$ of noncanonical candidate sites are removed, significantly more than A-to-G changes (Table 1). Next, we observed that both canonical and noncanonical sites are found clustered in the genome, an observation made also in previous large-scale studies (Bahn et al. 2011; Li et al. 2011). On average, almost half of the potential edited sites correspond to "dense sites," i.e., sites found very close to another candidate site (in this case, the length of the sequencing reads, $76 \mathrm{bp}$ ). While this clustering could result from the mechanism of action of the editing machinery, as is known to be the case for ADAR enzymes, mapping errors also generate such a pattern (Supplemental Fig. 5). It is worth noting that the clustering distance of most candidate sites depends on the length of the reads used for sequencing and the alignment method (Supplemental Fig. 1), suggesting that a large proportion of dense sites are most likely produced by mapping artifacts. Removing these sites from the analysis results in $91 \%$ of noncanonical sites filtered out, leaving A-to-G substitutions as the main change observed (Fig. 3, top left). However, three-quarters of the remaining sites are located in repetitive regions, where misalignments are particularly frequent (Brawand et al. 2011; Garber et al. 2011; Schrider et al. 2011; Treangen and Salzberg 2012). When only nonrepetitive regions are considered, a striking $98 \%$ of noncanonical candidate sites are filtered (Table 1; Fig. 3).

As for A-to-G changes, consistent with the accepted view of mammal RNA editing built over the last $20 \mathrm{yr}$ (Nishikura 2010), 94\% of the sites lie in repetitive regions of the genome. This is most likely the consequence of the biological activity of ADAR enzymes and not the result of a

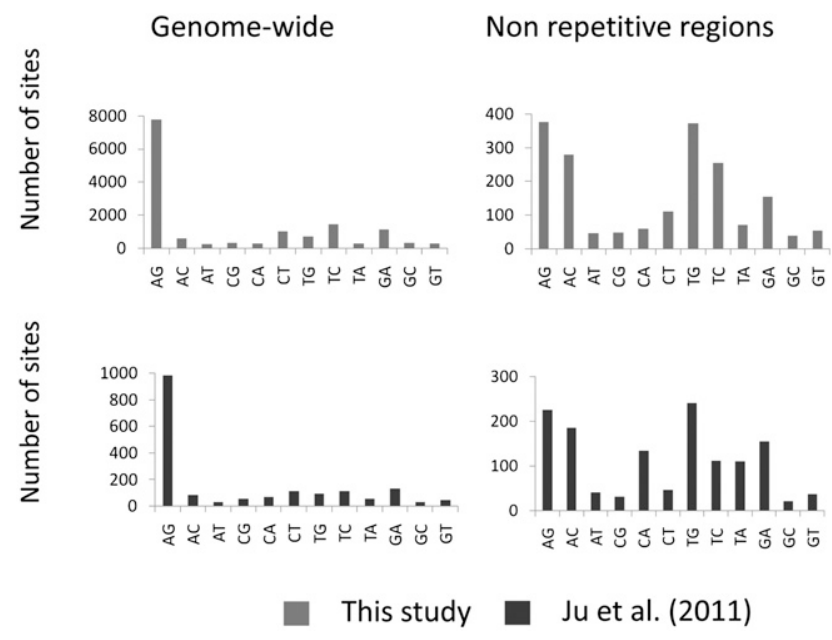

FIGURE 3. Recoding events identified by RNA-Seq. Number of events for the 12 types of differences between RNA sequence and genomic DNA sequence observed in lymphoblast cell transcriptomes. Labels of $x$-axis denote DNA and RNA nucleotides observed (e.g., "AG" stands for an A observed in the gDNA and a G observed in the cDNA). See Materials and Methods for details on the definition of repetitive regions. mapping artifact: the presence of A-to-G changes is not uniform across all types of repetitive regions, but occurs mostly in Alu sequences. When the length of the regions is taken into consideration, Alu regions show an impressive 25-fold enrichment of A-to-G changes with respect to other annotated repetitive regions. As a result, once the repetitive regions are removed from the analysis, the prevalence of A-to-G changes detected genome wide disappears (Fig. 3) and the total number of observed sites decreases drastically. Very similar patterns of nucleotide changes were observed in our data sets and the reported sites of $\mathrm{Ju}$ et al. (2011), despite the different approaches used (Fig. 3).

By applying a set of stringent criteria to avoid most artifacts of a high-throughput sequencing approach, we were able to identify between $85 \%$ and $98 \%$ of noncanonical editing sites as false positives. There are certainly other errors that we did not account for, and so the number of spurious mismatches may still be underestimated. But even if this is not the case, the remaining 1190 noncanonical sites represent a low rate of sequence change throughout the transcribed genome: approximately two edited bases per million bases transcribed, that is, in the same order of magnitude of the estimated error rate of RNA polymerases. Hence, if real, noncanonical recoding of RNA sequences does not seem to have a major quantitative effect on the transcriptome. In what follows, to better evaluate the overall impact of RNA editing on coding sequences, we will narrow the studied regions, focusing on nonrepetitive exons.

\section{Editing sites are rare in exons and coding regions}

In order to discard any effects specific to our methodological pipeline, we collected the sets of edited sites reported by previous studies based on high-throughput sequencing of human transcriptomes ( $\mathrm{Li}$ et al. 2009b, 2011; Bahn et al. 2011; Ju et al. 2011; Peng et al. 2012). In this way, different methods, experimental settings, and cell types are analyzed, so that the trends observed should not be specific to a particular condition. The following analyses were thus performed, when possible, in these data sets, in addition to the sites detected by our pipeline.

Table 2 shows the number of canonical A-to-G changes detected in large-scale approaches so far. In all but one study (Li et al. 2011), the distribution of sites across the regions is very consistent, showing few edited sites in coding regions: $30-87$ sites in all exons, and 12-28 sites in coding exons. This agreement is remarkable given the differences in cell types, number of samples, and methodological approaches used in these works. It should be noted that the data set reported by Li et al. (2011) has been shown to contain a very high number of false positives (Schrider et al. 2011; Kleinman and Majewski 2012; Lin et al. 2012; Pickrell et al. 2012), which explains the significantly 
TABLE 2. Number of canonical A-to-G editing sites reported in this and previous large-scale data sets, outside repetitive regions

\begin{tabular}{|c|c|c|c|c|c|c|}
\hline Study & Data set & Genome-wide & All exons & $5^{\prime}$ UTR & 3' UTR & Coding exons \\
\hline Bahn et al. (2011) & Breast cancer & 852 & 65 & 20 & 31 & 18 \\
\hline Bahn et al. (2011) & Glioblastome & 219 & 55 & 17 & 30 & 13 \\
\hline Li et al. (2009b) & Several cell types & 235 & 74 & 19 & 39 & 28 \\
\hline Li et al. (2009b), confident set & Several cell types & 121 & 50 & 12 & 28 & 20 \\
\hline Peng et al. (2012) & Lymphoblasts & 477 & 87 & 31 & 40 & 21 \\
\hline Ju et al. (2011) & Lymphoblasts & 225 & 30 & 4 & 18 & 12 \\
\hline This study & NA12891, Lymphoblast & 312 & 70 & 27 & 31 & 23 \\
\hline This study & NA12892, Lymphoblast & 311 & 65 & 23 & 37 & 14 \\
\hline Li et al. (2011) & Lymphoblasts & 2120 & 1935 & 277 & 1042 & 892 \\
\hline
\end{tabular}

higher number of editing sites reported and the completely different distribution of sites across regions that this data set displays for all of the analyses performed here. On the other hand, while the number of sites across regions is consistent across studies, the overlap of specific sites detected is almost nonexistent, even when the analyses are performed in the same cell type, as is the case for lymphoblastoid cell lines. The overlap of sites between pairs of data sets, when considering nonrepetitive regions genome wide, is $20 \%$ for the two biological replicates we studied, and $<3 \%$ in all other cases. In coding regions, no more than three common sites are found in any pair of reported data sets. This suggests that for most of the edited sites located in coding regions, RNA editing by ADAR1 is not essential for cell survival. To further validate these A-to-G sites by an independent method, we selected 15 sites in UTR and 12 sites in coding regions, obtained cDNA and gDNA from the same cell culture, and sequenced them with the Sanger method (Table 3). In all but one of the UTR sites sequenced, RNA editing was clearly seen in the chromatograms (Supplemental Fig. 2). Coding sites, on the other hand, show a much lower validation rate than UTR sites. Of all sites showing evidence of A-to-G editing in both samples, only four sites could be successfully amplified and sequenced, with two of them being true positives.

Next, we studied the distribution of sites for all sequence changes in nonrepetitive exons and coding exons (Fig. 4). The complete list of sites is provided in Supplemental Table 3. In the same way as A-to-G sites, the total number of noncanonical changes decreases sharply in these regions, by almost two orders of magnitude for most types of change.

TABLE 3. Candidate A-to-G editing sites validated by Sanger sequencing

\begin{tabular}{|c|c|c|c|c|c|c|}
\hline Gene & Description & Site & NA12891 & NA12892 & NA12891 & NA12892 \\
\hline FNLB & filamin $B, \beta$ (actin binding protein 278) & chr3:58116831 & 60 & 32 & 17 & 38 \\
\hline COG3 & component of golgi transport complex 3 & chr13:44988372 & 25 & 16 & 11 & 1 \\
\hline SRGAP2 & SLIT-ROBO Rho GTPase activating protein 2 & chr1:204686084 & 0 & 0 & 0 & 12 \\
\hline ANKFY1 & ankyrin repeat and FYVE domain containing 1 & chr17:4092417 & 0 & 0 & 23 & 0 \\
\hline POLR1B & RNA polymerase I polypeptide B isoform 1 & chr2:113050712 & 42 & 34 & 76 & 53 \\
\hline CASC5s01 & cancer susceptibility candidate 5 isoform 1 & chr15:38741917 & 27 & 28 & 21 & 13 \\
\hline GNL3L & guanine nucleotide binding protein-like 3 & chrX:54606499 & 39 & 37 & 18 & 22 \\
\hline FAM20B & hypothetical protein LOC9917 & chr1:177309528 & 32 & 31 & 22 & 23 \\
\hline PPIA & peptidylprolyl isomerase A & chr7:44807913 & 19 & - & 10 & 16 \\
\hline C22orf9 & hypothetical protein LOC23313 isoform b & chr22:43968528 & 59 & 42 & 37 & 38 \\
\hline PUM2 & pumilio homolog 2 & chr2:20314300 & 28 & 12 & 15 & 12 \\
\hline KIAA0621 & GTPase regulator & chr5:142585629 & 15 & 20 & 0 & 12 \\
\hline DDX58 & DEAD/H (Asp-Glu-Ala-Asp/His) box polypeptide & chr9:32446380 & 36 & 35 & 40 & 31 \\
\hline \multirow[t]{4}{*}{ TEP1 } & $\begin{array}{l}\text { Homo sapiens mRNA for Telomerase protein } \\
\text { component } 1 \text { variant protein }\end{array}$ & chr14:19905026 & 24 & 16 & 5 & 10 \\
\hline & & chr14:19904223 & 30 & 28 & 5 & 13 \\
\hline & & chr14:19904307 & 62 & 79 & 6 & 83 \\
\hline & & chr14:19904333 & 26 & 25 & 10 & 18 \\
\hline PCCMT & Prenylcysteine carboxyl methyltransferase & chr1:6205174 & 49 & 32 & 9 & 28 \\
\hline ZBTB43 & zinc finger and BTB domain containing 43 & chr9:128607209 & 0 & 0 & 13 & 29 \\
\hline
\end{tabular}




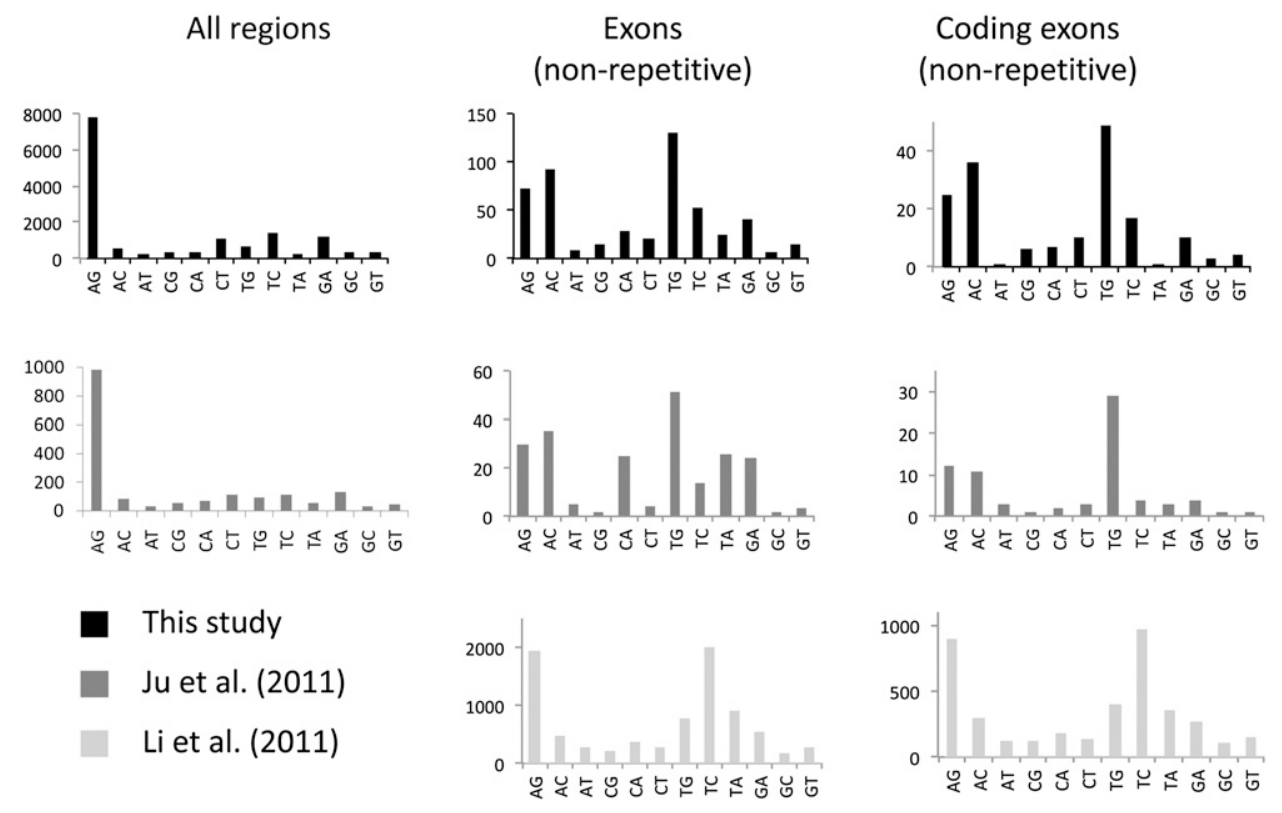

FIGURE 4. Recoding events across genomic regions. Number of events observed for the 12 types of differences between RNA sequence and genomic DNA sequence, separated by type of region. See Materials and Methods for details on the definition of repetitive regions.

Note that the distributions obtained in our samples and the data set reported by Ju et al.(2011) are remarkably similar, as observed before. In coding regions, only two types of change remain relatively frequent: $\mathrm{T}-$ to- $\mathrm{G}$ and A-to-C, outnumbering the canonical editing sites consistently across our data set and the data set of Ju et al. (2011).

In order to further understand the nature of these noncanonical changes and discard potential sequence-specific errors still undetected, we performed a sequence analysis of regions flanking editing sites. For each type of change and each data set, we extracted 50 nt upstream of and downstream from all candidate editing sites (genome wide) and constructed sequence logos (Crooks et al. 2004) with the resulting alignments. For all three data sets, we found a single specific sequence context surrounding T-to-G and A-to-G sites (Fig. 5; Supplemental Fig. 3). This consensus sequence corresponds to a motif previously shown to induce sequence-specific errors in Illumina sequencers (Meacham et al. 2011). Note that both T-to-G and A-to-C mismatches originate in the same sequence motif, since they correspond in fact to complementary cases: a T-to-G change in one strand of the cDNA corresponds to an A-to- $\mathrm{C}$ change in the reverse strand. The surrounding sequence context found is therefore the same (reverse complemented). Consistently, most of these sites were supported by unidirectional reads, where the reads presenting a mismatch are the ones that find the motif immediately upstream of the edited site (Supplemental Fig. 4). It should be noted that these sites went undetected in the corresponding filtering step of the pipeline due to the small number of alternative reads they present, which prevents us from effectively applying a filter based on the directionality of the reads. Altogether, this evidence indicates that both A-to-G and T-to-G mismatches, the only noncanonical changes we find relatively frequently in coding regions, originate in the elongation step of RNA sequencing and are unlikely to be the product of a true editing event.

\section{DISCUSSION}

In this work we studied the extent of recoding of RNA molecules in the transcriptome of human lymphoblasts, a cell type expressing ADAR1 protein at high levels. We first presented a sequencing approach to detect editing sites in human cells, and subsequently compared the results obtained on these samples to the sites reported in previous large-scale approaches, which we reanalyzed with a particular emphasis on protein-coding sequences. The approach presented here is general and can be applied in the future to other cell types and conditions. For example, a similar analysis can be performed using cells of neural origin in order to quantitatively assess the impact of editing by ADAR2 genome wide.

For canonical A-to-G RNA editing, the results obtained in this and previous large-scale studies recapitulate what has been the consensus view built over $20 \mathrm{yr}$ of biological experiments: this is a relatively frequent, widespread process throughout the human genome, but occurs almost exclusively in repetitive and noncoding regions. The biological function of RNA editing in mammals is not yet fully understood. We know that this mechanism is essential for a number of important brain-specific coding transcripts, where the resulting sequence change modifies protein 


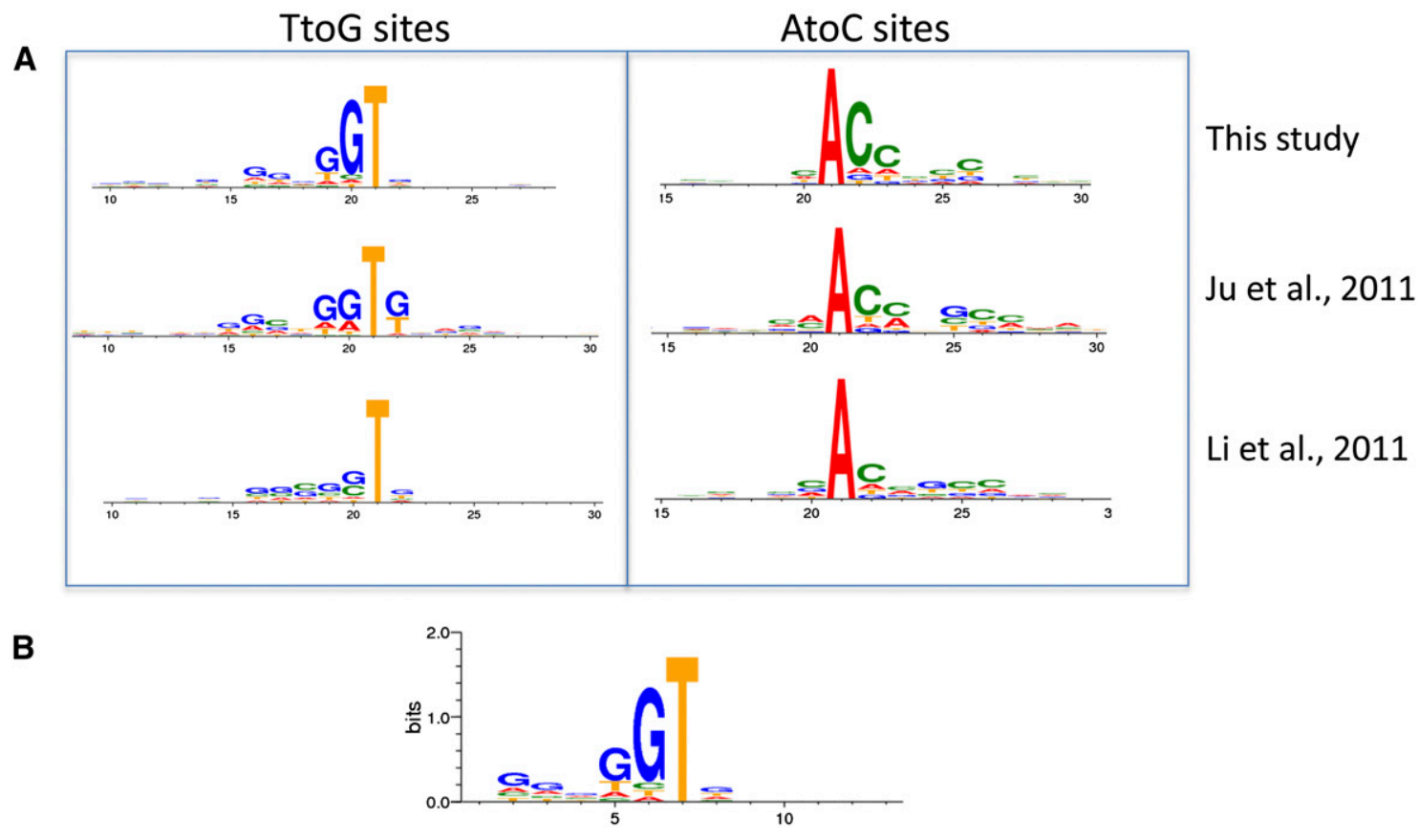

FIGURE 5. (A) Sequence logos (Crooks et al. 2004) generated using sequences flanking candidate recoded sites. (B) A single motif is found when sites corresponding to the same case in the cDNA molecule are grouped together (see Materials and Methods for details); T-to-G and the reverse complement of A-to-G flanking regions are shown.

function substantially (Pullirsch and Jantsch 2010). Indeed, ADAR deficiencies lead to several abnormal phenotypes, including seizures and death in ADAR2 null mice (Higuchi et al. 2000) and embryonic-lethal phenotypes in ADAR1deficient mice (Wang et al. 2000; Hartner et al. 2004). In spite of this, our results point to a reduced quantitative impact of RNA editing in diversifying the proteome, at least in the cell types studied here. First, the number of recoding events in protein sequences is relatively small, considering that we are assessing RNA editing transcriptome-wide, with a very sensitive sequencing strategy. Moreover, there is little or no overlap of editing sites across studies and biological replicates. The small overlap observed in different cell types is not surprising, since RNA editing is known to be a tissuespecific process. We see in addition, however, little consistency for sites detected in different biological replicates of the same tissue, suggesting that for most sites constitutive editing by ADAR1 is not essential for survival of the cell. Instead, the genomic distribution of edited sites observed both in our samples and previous large-scale sequencing studies support a primary role of ADAR1 as a defense system against elements potentially damaging to the genome. Alu sequences are 25 times more frequently edited than other repetitive regions. It has been previously shown that in many cells mRNA containing Alu repeats are retained in the nucleus to avoid translation, with nuclear retention correlating with A-to-G editing (Chen et al. 2008). Along these lines, the interferon-inducible deaminase ADAR1 has been frequently proposed to be one of the antiviral effector proteins within the innate immune response (Hamilton et al. 2010; Samuel 2012). In this case, editing of host transcripts would be produced mainly as a side effect of this activity. The occurrence of editing sites almost exclusively in noncoding repetitive regions is consistent with this hypothesis.

As for noncanonical sites, on the other hand, the scenario is very different. We provided evidence that noncanonical RNA editing is unlikely to be a true biological process. Although we cannot completely rule out the possibility of rare noncanonical recoding events, we did not find any evidence pointing to a functional or regulatory role associated with them. Genome wide, the sites remaining after filtering out false positives are rare, even more so in coding regions, a result consistent with the findings in the work of Ramaswami et al. (2012). We showed that the most frequent ones (T-to-G and A-to-G) originate in a sequencespecific error previously described. We also observe some $\mathrm{T}$-to- $\mathrm{C}$ changes in coding regions in our samples, which are less frequent in the reported sites of Ju et al. (2011), and that are not explained by any specific sequencing error. These sites most probably originate in a canonical A-to-G RNA-editing event occurring in the opposite strand to the annotated gene. These events are incorrectly interpreted as T-to-C changes, since strand information is lost during RNA sequencing as performed here. Incorrect assignment of gene strand may originate in errors present in current annotations, but it can also happen when antisense transcription occurs, which has been recently shown to be very 
frequent in the mammal transcriptome (RIKEN Genome Exploration Research Group and Genome Science Group and the Fantom Consortium et al. 2005). Consistently, Bahn et al. (2011) reported a decrease in T-to-C changes when $A D A R 1$ is knocked down, indicating that a significant proportion of these sites may actually be produced by canonical A-to-G RNA editing.

In this work we show that RNA editing can be detected using a small number of samples by high-throughput sequencing, when technical artifacts are controlled for. We characterized a large number of sequencing and mapping errors associated with an RNA-Seq approach. Mapping of reads spanning splicing junctions and multicopy regions of the genome, as well as genomic locations bearing specific sequence motifs represented important challenges. As sequencing technology and analysis methods improve, these and other types of systematic errors will probably be reduced, allowing us to gain further insights into the biological importance of this process. However, at the present time we caution against assigning too much biological significance to the interpretation of highly noise-prone massively parallel sequencing data.

\section{MATERIALS AND METHODS}

\section{Gene annotations and software}

Genomic coordinates of genes, exonic regions, exon-exon junctions, RepeatMasker regions, and segmental duplications were defined based on the UCSC genome annotation (Rhead et al. 2010). Genomic locations of dbSNP 132 records in hg18 coordinates were obtained with ANNOVAR (Wang et al. 2010). Integrated Genome Viewer (http://broadinstitute.org/igv) was used to visualize the data. BEDTools (Quinlan and Hall 2010) and custom scripts were used to compute the number of sites and overlaps between data sets.

\section{Samples}

For the identification of editing sites, we used deep RNA-Seq data generated on two unrelated HapMap CEU lymphoblast cell lines (NA12891 and NA12892), reported in Lalonde et al. (2011). This data consisted of paired-end, 76-bp reads obtained using the Illumina GAIIx technology. Reads were mapped to the human reference genome hg18 using TopHat (Trapnell et al. 2009) coupled to Bowtie (Langmead et al. 2009). Reads mapping to multiple locations were discarded. We obtained high-coverage genomic data $(20-60 \times)$ for the corresponding individuals from The 1000 Genomes Project pilot 2 (Altshuler et al. 2010).

\section{Repetitive regions}

Repetitive regions of the genome where short sequencing reads may have ambiguous mapping were defined by combining two sets of intervals. First, we used UCSC annotations: RepeatMasker, simple repeats, and segmental duplications. Second, additional multicopy regions were identified by aligning every 76-nt exonic window of the reference human genome against itself with BWA
(Li and Durbin 2010), and recording those that map to multiple locations. We combined these two data sets and extended the boundaries of the resulting intervals by $10 \mathrm{nt}$ in order to include the immediate flanking regions, which are also prone to alignment errors (Kleinman and Majewski 2012). The final interval set is provided as Supplemental Material. The resulting set of intervals is referred throughout the main text as "repetitive regions."

\section{Detection of intronless gene duplications}

We simulated all 76-nt-long reads spanning splice junctions with at least $15 \mathrm{nt}$ covering each exon. The algorithm was as follows: for each possible splice junction of genes based on UCSC annotations, the reference sequence corresponding to the upstream and downstream exon was extracted in a sliding window of $76 \mathrm{nt}$, with a sliding step of $1 \mathrm{nt}$, and the coordinates of the original location were recorded. A minimum overhang of $15 \mathrm{nt}$ was required in this step. Reads were then mapped back to the reference genome with Bowtie (Langmead et al. 2009), a nonspliced aligner, with the same settings used for the alignment of the RNAseq data, allowing for up to two mismatches. The regions where reads mapped uniquely without indels (in a location different from the originating location) were interpreted as intronless gene duplications. Overlapping segments were combined into longer stretches, and the boundaries of the resulting intervals were extended by $200 \mathrm{nt}$ to include the inferred flanking exon. The final interval set is provided as Supplemental Material. The analysis was also performed using BWA (Altshuler et al. 2010) for the alignment step, with read lengths of 76 and $100 \mathrm{nt}$, with similar results (data not shown).

\section{RNA editing screening}

First, base calls were made using SAMtools ( $\mathrm{Li}$ et al. 2009a) both for DNA and RNA sequences, using the human genome hg18 as reference. Sites where RNA sequence differed from the DNA sequence were regarded as candidate-editing sites, when the mismatch was supported by at least two reads and $5 \%$ of the total reads observed at that position. Base calls were then re-evaluated, excluding from further analysis sites not complying with the following criteria: (1) minimum sequencing coverage (four reads for RNA, 10 reads for DNA), (2) Phred-like score greater or equal to 20, (3) average mapping quality greater or equal to 15 , (4) no insertion or deletion with respect to the reference genome, (5) total number of genomic reads less than a certain cutoff (here, 1000 reads) to exclude potential PCR duplicates, (6) consensus score for DNA sequencing data greater or equal to 70 ; if lower, no presence of alternative base in sequencing reads.

A second set of filters was then applied to deal with genomic regions prone to sequencing and mapping errors. First, sites located in unmappable segments (multicopy regions, see above) or intronless gene duplications (see above) were filtered out. Sites where the alternative base was present only in terminal read positions (first or last bases of a sequencing read) were also filtered out, as well as those only supported by unidirectional reads (i.e., reads sequencing exclusively one strand of the cDNA). The latter filter was only applied when the total number of alternative reads exceeded a defined threshold (here, three reads) to avoid a high number of false negatives. Finally, intronic sites located within $6 \mathrm{bp}$ of exon boundaries of UCSC genes and sites present in dbSNP 132 were also removed. 


\section{Validation of editing sites by Sanger sequencing}

Lymphoblastoid cell lines (LCL) were cultured in RPMI 1640 media with $2 \mathrm{mM}$ L-glutamine, $15 \%$ fetal bovine serum, and $1 \%$ penicillin-streptomycin at $37^{\circ} \mathrm{C}$ with $5 \% \mathrm{CO}_{2}$. RNA was extracted using TRIzol (Invitrogen) and quality assessed by Agilent 2100 BioAnalyzer (Agilent Technologies). High-quality RNA was used to synthesize first-strand cDNA with $500 \mathrm{ng}$ of random hexamers (Invitrogen) and Superscript II reverse transcriptase (Invitrogen) according to manufacturer's instructions. DNA was successfully extracted from cell lysates of LCL and resuspended in $200 \mu \mathrm{L}$ of PBS using the GenElute DNA Miniprep Kit (SigmaAldrich).

Primer pairs for PCR amplification and sequencing of each editing site were generated using Primer3Plus (Untergasser et al. 2007). Primers were designed to obtain a product of $\sim 300 \mathrm{bp}$, which was considered optimal for amplification, purification, and sequencing, with the editing site in the center portion of the fragment. To minimize amplification of homologous genomic sequences, primer pairs were filtered using both the UCSC In Silico PCR software and NCBI Primer-Blast against Refseq mRNA. Only pairs yielding a single predicted product were used. PCR reactions were performed in triplicate and purified prior sequencing using Exo-SAP PCR cleanup protocol as recommended by the manufacturer (Affymetrix). Products were sequenced by conventional Sanger methods (Applied Biosystems) and purified by ethanol precipitation prior to obtaining DNA chromatograms in a 3730xl DNA Analyzer. Sequence chromatograms were aligned and analyzed with PeakPeaker v.2.0 (Ge et al. 2005) with the default settings to quantify the relative amount of the two alternative bases measured from the chromatogram after peak intensity normalization.

\section{Sequence motif analysis}

Flanking sequences of candidate editing sites located in annotated UCSC genes (where strand information can be retrieved) were extracted from the reference genome using BEDTools (Quinlan and Hall 2010). For each editing type, all 40-nt-long flanking sequences were used to build a sequence logo with WebLogo (Crooks et al. 2004). Alternatively, sequence logos were built grouping together editing types that correspond to the same case in a double-stranded cDNA molecule, e.g., T-to-G(+)/A-to-G(-) and the reverse complement of A-to-G(+)/T-to-G(-), where $(+)$ and $(-)$ stand for the strand where the gene containing the candidate site is located.

\section{SUPPLEMENTAL MATERIAL}

Supplemental material is available for this article.

\section{ACKNOWLEDGMENTS}

We thank Santiago Costantino for useful discussions. This work was supported by grants from Genome Quebec/Genome Canada (to V.A.); the Canadian Institutes of Health Research and Canada Research Chairs (to J.M.); and the Fonds de recherche du Québec-Santé (to C.L.K.).

Author contributions: C.L.K. and J.M. conceived the study, performed data analyses, and drafted the manuscript. V.A. performed experimental work. All authors read and approved the final manuscript.

Received April 27, 2012; accepted June 19, 2012.

\section{REFERENCES}

Altshuler DL, Durbin RM, Abecasis GR, Bentley DR, Chakravarti A, Clark AG, Collins FS, De La Vega FM, Donnelly P, Egholm M, et al. 2010. A map of human genome variation from populationscale sequencing. Nature 467: 1061-1073.

Bahn JH, Lee JH, Li G, Greer C, Peng G, Xiao X. 2011. Accurate identification of A-to-I RNA editing in human by transcriptome sequencing. Genome Res 22: 142-150.

Bass BL. 2002. RNA editing by adenosine deaminases that act on RNA. Annu Rev Biochem 71: 817-846.

Bhat GJ, Koslowsky DJ, Feagin JE, Smiley BL, Stuart K. 1990. An extensively edited mitochondrial transcript in kinetoplastids encodes a protein homologous to ATPase subunit 6. Cell 61: 885-894.

Brawand D, Soumillon M, Necsulea A, Julien P, Csardi G, Harrigan P, Weier M, Liechti A, Aximu-Petri A, Kircher M, et al. 2011. The evolution of gene expression levels in mammalian organs. Nature 478: $343-348$.

Burns CM, Chu H, Rueter SM, Hutchinson LK, Canton H, Sanders-Bush E, Emeson RB. 1997. Regulation of serotonin-2C receptor G-protein coupling by RNA editing. Nature 387: 303-308.

Chen L-L, DeCerbo JN, Carmichael GG. 2008. Alu element-mediated gene silencing. EMBO J 27: 1694-1705.

Crooks GE, Hon G, Chandonia J-M, Brenner SE. 2004. WebLogo: A sequence logo generator. Genome Res 14: 1188-1190.

Feagin JE, Abraham JM, Stuart K. 1988. Extensive editing of the cytochrome c oxidase III transcript in Trypanosoma brucei. Cell 53: 413-422.

Garber M, Grabherr MG, Guttman M, Trapnell C. 2011. Computational methods for transcriptome annotation and quantification using RNA-seq. Nat Methods 8: 469-477.

Ge B, Gurd S, Gaudin T, Dore C, Lepage P, Harmsen E, Hudson TJ, Pastinen T. 2005. Survey of allelic expression using EST mining. Genome Res 15: 1584-1591.

George CX, Samuel CE. 1999. Human RNA-specific adenosine deaminase ADAR1 transcripts possess alternative exon 1 structures that initiate from different promoters, one constitutively active and the other interferon inducible. Proc Natl Acad Sci 96: 46214626.

Hamilton CE, Papavasiliou FN, Rosenberg BR. 2010. Diverse functions for DNA and RNA editing in the immune system. RNA Biol 7: 220-228.

Hartner JC, Schmittwolf C, Kispert A, Müller AM, Higuchi M, Seeburg PH. 2004. Liver disintegration in the mouse embryo caused by deficiency in the RNA-editing enzyme ADAR1. J Biol Chem 279: 4894-4902.

Higuchi M, Maas S, Single FN, Hartner J, Rozov A, Burnashev N, Feldmeyer D, Sprengel R, Seeburg PH. 2000. Point mutation in an AMPA receptor gene rescues lethality in mice deficient in the RNA-editing enzyme ADAR2. Nature 406: 78-81.

Horsch M, Seeburg PH, Adler T, Aguilar-Pimentel JA, Becker L, Calzada-Wack J, Garrett L, Gotz A, Hans W, Higuchi M, et al. 2011. Requirement of the RNA-editing enzyme ADAR2 for normal physiology in mice. J Biol Chem 286: 18614-18622.

Huang H, Tan Bao Z, Shen Y, Tao J, Jiang F, Sung Ying Y, Ng Choon K, Raida M, Köhr G, Higuchi M, et al. 2012. RNA editing of the IQ domain in $\mathrm{Ca}_{\mathrm{v}} 1.3$ channels modulates their $\mathrm{Ca}^{2+}$-dependent inactivation. Neuron 73: 304-316.

Ju YS, Kim J-I, Kim S, Hong D, Park H, Shin J-Y, Lee S, Lee W-C, Kim S, Yu S-B, et al. 2011. Extensive genomic and transcriptional diversity identified through massively parallel DNA and RNA sequencing of eighteen Korean individuals. Nat Genet 43: 745-752. 
Kleinman CL, Majewski J. 2012. Comment on "Widespread RNA and DNA Sequence Differences in the Human Transcriptome". Science 335: 1302. doi: $10.1126 /$ science. 1209658 .

Knoop V. 2010. When you can't trust the DNA: RNA editing changes transcript sequences. Cell Mol Life Sci 68: 567-586.

Lalonde E, Ha KCH, Wang Z, Bemmo A, Kleinman CL, Kwan T, Pastinen T, Majewski J. 2011. RNA sequencing reveals the role of splicing polymorphisms in regulating human gene expression. Genome Res 21: 545-554.

Langmead B, Trapnell C, Pop M, Salzberg SL. 2009. Ultrafast and memory-efficient alignment of short DNA sequences to the human genome. Genome Biol 10: R25. doi: 10.1186/gb-2009-103-r25.

Levanon EY, Eisenberg E, Yelin R, Nemzer S, Hallegger M, Shemesh R, Fligelman ZY, Shoshan A, Pollock SR, Sztybel D, et al. 2004. Systematic identification of abundant A-to-I editing sites in the human transcriptome. Nat Biotechnol 22: 1001-1005.

Li H, Durbin R. 2010. Fast and accurate long-read alignment with Burrows-Wheeler transform. Bioinformatics 26: 589-595.

Li H, Handsaker B, Wysoker A, Fennell T, Ruan J, Homer N, Marth G, Abecasis G, Durbin R, Subgroup GPDP. 2009a. The Sequence Alignment/Map format and SAMtools. Bioinformatics 25: 20782079.

Li JB, Levanon EY, Yoon JK, Aach J, Xie B, LeProust E, Zhang K, Gao Y, Church GM. 2009b. Genome-wide identification of human RNA editing sites by parallel DNA capturing and sequencing. Science 324: 1210-1213.

Li M, Wang IX, Li Y, Bruzel A, Richards AL, Toung JM, Cheung VG. 2011. Widespread RNA and DNA sequence differences in the human transcriptome. Science 333: 53-58.

Lin W, Piskol R, Tan MH, Li JB. 2012. Comment on "Widespread RNA and DNA sequence differences in the human transcriptome". Science 335: 1302. doi: 10.1126/science.1210624.

Lomeli H, Mosbacher J, Melcher T, Hoger T, Geiger, Kuner T, Monyer H, Higuchi M, Bach A, Seeburg P. 1994. Control of kinetic properties of AMPA receptor channels by nuclear RNA editing. Science 266: 1709-1713.

Meacham F, Boffelli D, Dhahbi J, Martin D, Singer M, Pachter L. 2011. Identification and correction of systematic error in highthroughput sequence data. BMC Bioinformatics 12: 451. doi: 10.1186/1471-2105-12-451.

Nakamura K, Oshima T, Morimoto T, Ikeda S, Yoshikawa H, Shiwa Y, Ishikawa S, Linak MC, Hirai A, Takahashi H, et al. 2011. Sequence-specific error profile of Illumina sequencers. Nucleic Acids Res 39: e90. doi: 10.1093/nar/gkr344.

Nishikura K. 2010. Functions and regulation of RNA editing by ADAR deaminases. Annu Rev Biochem 79: 321-349.

Nishimoto Y, Yamashita T, Hideyama T, Tsuji S, Suzuki N, Kwak S. 2008. Determination of editors at the novel A-to-I editing positions. Neurosci Res 61: 201-206.

The 1000 Genomes Project Consortium. 2010. A map of human genome variation from population-scale sequencing. Nature 467: 1061-1073.
Peng Z, Cheng Y, Tan BC-M, Kang L, Tian Z, Zhu Y, Zhang W, Liang $\mathrm{Y}, \mathrm{Hu} \mathrm{X}$, Tan X, et al. 2012. Comprehensive analysis of RNA-Seq data reveals extensive RNA editing in a human transcriptome. Nat Biotechnol 30: 253-260.

Pickrell JK, Gilad Y, Pritchard JK. 2012. Comment on "widespread RNA and DNA sequence differences in the human transcriptome". Science 335: 1302. doi: 10.1126/science.1210484.

Pullirsch D, Jantsch MF. 2010. Proteome diversification by adenosine to inosine RNA-editing. RNA Biol 7: 205-212.

Quinlan AR, Hall IM. 2010. BEDTools: A flexible suite of utilities for comparing genomic features. Bioinformatics 26: 841-842.

Ramaswami G, Lin W, Piskol R, Tan MH, Davis C, Li JB. 2012. Accurate identification of human Alu and non-Alu RNA editing sites. Nat Meth doi: 10.1038/nmeth.1982.

Rhead B, Karolchik D, Kuhn RM, Hinrichs AS, Zweig AS, Fujita PA, Diekhans M, Smith KE, Rosenbloom KR, Raney BJ, et al. 2010. The UCSC Genome Browser database: Update 2010. Nucleic Acids Res 38: D613-D619.

Riedmann EM, Schopoff S, Hartner JC, Jantsch MF. 2008. Specificity of ADAR-mediated RNA editing in newly identified targets. RNA 14: $1110-1118$.

RIKEN Genome Exploration Research Group and Genome Science Group and the Fantom Consortium, Katayama S, Tomaru Y, Kasukawa T, Waki K, Nakanishi M, Nakamura M, Nishida H, Yap CC, Suzuki M, Kawai J, et al. 2005. Antisense transcription in the mammalian transcriptome. Science 309: 1564-1566.

Rosenberg BR, Hamilton CE, Mwangi MM, Dewell S, Papavasiliou FN. 2011. Transcriptome-wide sequencing reveals numerous APOBEC1 mRNA-editing targets in transcript 3' UTRs. Nat Struct Mol Biol 18: 230-236.

Samuel CE. 2012. Adenosine deaminases acting on RNA (ADARs) and A-to-I editing. Vol. 353 (ed. CE Samuel), pp. 163-195. Springer, Berlin, Heidelberg, Germany.

Schrider DR, Gout J-F, Hahn MW. 2011. Very few RNA and DNA sequence differences in the human transcriptome. PLOS ONE 6: e25842. doi: 10.1371/journal.pone.0025842.

Sommer B, Köhler M, Sprengel R, Seeburg PH. 1991. RNA editing in brain controls a determinant of ion flow in glutamate-gated channels. Cell 67: 11-19.

Trapnell C, Pachter L, Salzberg SL. 2009. TopHat: Discovering splice junctions with RNA-Seq. Bioinformatics 25: 1105-1111.

Treangen TJ, Salzberg SL. 2012. Repetitive DNA and next-generation sequencing: Computational challenges and solutions. Nat Rev Genet 13: 36-46.

Untergasser A, Nijveen H, Rao X, Bisseling T, Geurts R, Leunissen JAM. 2007. Primer3Plus, an enhanced web interface to Primer3. Nucleic Acids Res 35: W71-W74.

Wang Q, Khillan J, Gadue P, Nishikura K. 2000. Requirement of the RNA editing deaminase ADAR1 gene for embryonic erythropoiesis. Science 290: 1765-1768.

Wang K, Li M, Hakonarson H. 2010. ANNOVAR: Functional annotation of genetic variants from high-throughput sequencing data. Nucleic Acids Res 38: e164. doi: 10.1093/nar/gkg603. 

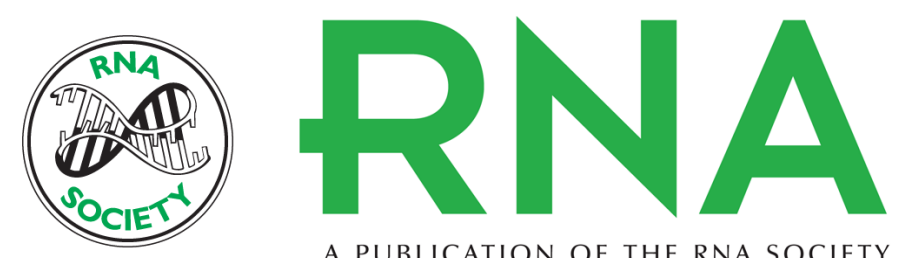

A PUBLICATION OF THE RNA SOCIETY

\section{RNA editing of protein sequences: A rare event in human transcriptomes}

Claudia L. Kleinman, Véronique Adoue and Jacek Majewski

RNA 2012 18: 1586-1596 originally published online July 25, 2012

Access the most recent version at doi:10.1261/rna.033233.112

\section{Supplemental http://rnajournal.cshlp.org/content/suppl/2012/07/10/rna.033233.112.DC1 Material}

References This article cites 49 articles, 15 of which can be accessed free at: http://rnajournal.cshlp.org/content/18/9/1586.full.html\#ref-list-1

\section{License}

Email Alerting Receive free email alerts when new articles cite this article - sign up in the box at the Service top right corner of the article or click here. 